\title{
SPH-DEM COUPLING FOR DEBRIS FLOWS
}

\section{MARIO GERMÁN TRUJILLO-VELA ${ }^{1,2}$, SERGIO ANDRÉS GALINDO-TORRES ${ }^{1 *}$, XUE ZHANG ${ }^{3}$, ALFONSO MARIANO RAMOS-CAÑÓN ${ }^{2}$ AND JORGE ALBERTO ESCOBAR-VARGAS ${ }^{2}$}

\author{
${ }^{1}$ Westlake University \\ No.18 Shilongshan Road, Xihu District, Hangzhou, Zhejiang Province, China \\ s.torreswestlake.edu.cn \\ 2 Pontificia Universidad Javeriana \\ Cra. 7 No. 40-62, Bogotá D.C., Colombia \\ ${ }^{3}$ University of Liverpool \\ Liverpool L69 3BX, United Kingdom
}

Key words: SPH-DEM coupling, three-phases model, debris flows simulation

\begin{abstract}
Debris flows are natural events with a high potential of damage due to the materials, volume, and velocity they can reach once the flows were triggered. Mathematical models and numerical schemes constitute a transcendental way to get a deeper comprehension of these natural phenomena. Thus, the coupling of numerical methods is becoming more relevant to describe the behaviour of debris flows. The coupling of Smooth Particle Hydrodynamics (SPH) and Discrete Element Method (DEM) is presented in this work to show the capability to represent the interaction of several materials simultaneously. SPH is employed to represent the fluid and soil by using different constitutive models, from a continuum approach. On the other hand, DEM describes immersed objects to represent large boulders and unmoveable boundary conditions. Thus, it is possible to couple the behaviour occurring at very different scales, fines and water through the continuum approach, and boulders with the discrete one. A hypothetical case here presented shows the potential of our coupling method for simulating debris flows.
\end{abstract}

\section{INTRODUCTION}

Debris flows are mixtures of water, soil, and large boulders that descend the basins and destroy everything on their path. The study of such phenomena is crucial for hazard assessment and mitigation plans. That is why there is considerable interest in developing mathematical models and numerical techniques to obtain suitable results in respect to debris flows. The improvement of the computational resources and numerical methods has allowed increasing the complexity of modelling these phenomena, adding the third dimension, more phases and the interaction with obstacles [1, 2]. Hence, meshless approaches have been gaining importance. These techniques also allow handling complex geometries, interaction with several methods and materials in a more natural manner. For example, Smooth Particle Hydrodynamics, SPH henceforth, has been employed to model many cases in soil mechanics, and fluid mechanics $[3,4$, $5,6,7]$. 
SPH has been coupled with other techniques such as Discrete Element Method, DEM henceforth, to represent the interaction with solids $[8,9,10,11]$. These two methods were developed to tackle problems at different scales: SPH to represent large scales directly by using constitutive laws, and DEM to obtain the general behaviour through the implementation of interaction laws in a small scale of granular assemblies $[12,13]$. Nevertheless, DEM can represent big objects with complex shapes and be useful to set up boundary conditions, fluid-structures, fluid-soils and fluid-soil-structures interaction problems.

[8] included rigid bodies into a fluid flow where the discretisation of the solids with SPH particles is still needed. [9] have coupled DEM to the traditional equations that describe the flow in porous media, where the mass conservation equation and the Darcy law are combined, neglecting the momentum equation. An analytical expression gives the interaction force after integrating the pressure on the surface of the spheres. Such pressure is computed by interpolating the variable from the established grid [9]. [10] implemented an algorithm to represent polyhedral DEM particles coupled with fluid SPH particles, where the normal force is based on the fluid pressure. [11] modelled rigid and deformable structures that interact with soil SPH particles, which normal force is based on a penetration method.

Also, [14] and [15] represented the soil employing DEM particles and the water using SPH particles, to reproduce landslide-induced waves where the SPH-DEM coupling is based on a drag force term, which involves an empirical formula. Other methods such as Finite Volumes Method (FVM), Finite Elements Method (FEM), Material Point Method (MPM) and Lattice Boltzmann Method (LBM) have been coupled to DEM to predict the interaction of debris flows with moving and flexible barriers [2, 16, 17]. To model large-deformation problems using mesh-based methods (i.g., FVM, FEM and LBM)

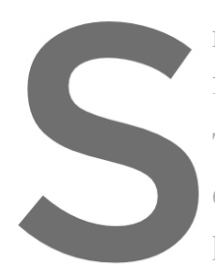
requires re-meshing, meshing ar with me flow in specific time-steps, or additional trentinents on the free surface of the flow. This work aims to devetch dimensional three-phases phases through the continu
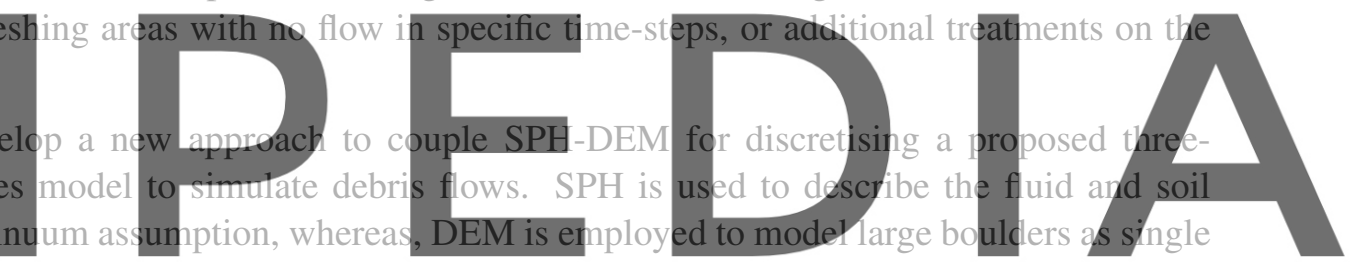

objects and the boundary conditions with the sphero-polyhedra approach as presented by $[18,13]$. The

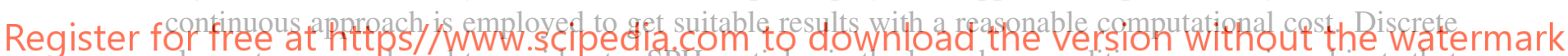
elements are employed to avoid extra SPH particles in the boundary conditions or moving objects that interact with the fluid or soil.

\section{NUMERICAL DISCRETISATION}

The conservation equations used to represent the fluid were discretised using the Weakly Compressible (WCSPH) approach. Thus the mass conservation and momentum equations are written as follows,

$$
\frac{D \rho_{i}}{D t}=\rho_{i} \sum_{j=1}^{n} \frac{m_{j}}{\rho_{j}} \mathbf{u}_{i j} \cdot \nabla_{i} W\left(r_{i j}, h\right)+D_{\delta}
$$




$$
\begin{aligned}
\frac{D \mathbf{u}_{i}}{D t}=\mathbf{g} & -\sum_{j=1}^{n} m_{j}\left(\frac{p_{i}}{\rho_{i}^{2}}+\frac{p_{j}}{\rho_{j}^{2}}+R_{T}+\Pi_{i j}\right) \nabla_{i} W\left(r_{i j}, h\right) \\
& +\sum_{j=1}^{n} 4 m_{j} \frac{\left(\mu_{i}+\mu_{j}\right)}{\left(\rho_{i}+\rho_{j}\right)^{2}} \cdot \mathbf{u}_{i j} \nabla_{i} W\left(r_{i j}, h\right)-\mathbf{a}_{i}^{f s}+\frac{\mathbf{F}_{i}^{f N}}{m_{i}}
\end{aligned}
$$

where the subindex $i$ and $j$ denote the point in the matter and the surrounding points, respectively. $n$ is the number of neighbouring particles. $\mathbf{u}_{i j}=\mathbf{u}_{i}-\mathbf{u}_{j}$ is the difference of the velocity between the two particles $i$ and $j, \mathbf{x}_{i j}=\mathbf{x}_{i}-\mathbf{x}_{j}$ is the vector that contains the distance between the two particles, $m$ is the mass, $\rho$ represents the density, $p$ is the thermodynamic pressure [19], and $\mathbf{g}$ is the gravity. $W\left(r_{i j}, h\right)$ the interpolating kernel, $\nabla_{i}$ denotes the gradient of the kernel taken with respect to the coordinates of particle $i$. The second term in Equation 1 is diffusive known as $\delta$-SPH [20], which is employed to eliminate the noise in the pressure field. $\Pi_{i j}$ is the artificial viscosity employed solely when shock wave phenomena are going to be treated, which is presented in detail in [21, 7]. The second and third terms on the righthand side of Equation 2 were discretised such as proposed by [21, 22] to handle discontinuities. $\mathbf{a}_{i}^{f s}$ represents the acceleration coming from forces due to the soil particles. $\mathbf{F}_{i}^{f N}=\mathbf{F}_{i(n)}^{f N}+\mathbf{F}_{i(\tau)}^{f N}$ is the net exerted force on the fluid particle by DEM objects, which is explained below Equation 13.

The mass conservation of soil is the same as Equation 1 without dissipative term, whereas the conservation of momentum is described such as,
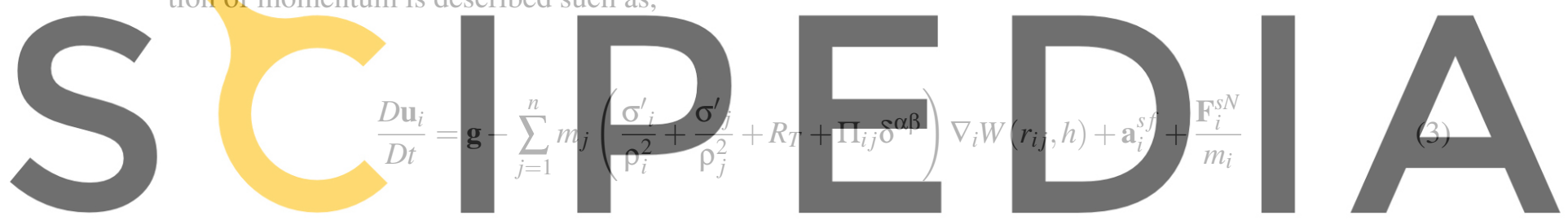

where $\sigma_{i}^{\prime}$ is the effective stress tensor that is computed using elastic perfectly-plastic model with the

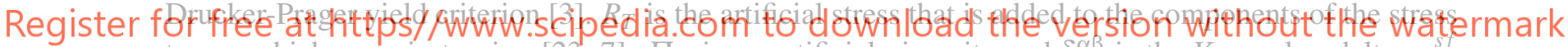
tensor which were in tension $[23,7]$. $\Pi_{i j}$ is an artificial viscosity and $\delta^{\alpha \beta}$ is the Kronecker delta. $\mathbf{a}_{i}$ represents the acceleration coming from forces due to the fluid particles. $\mathbb{F}_{i}^{s N}=\mathbb{F}_{i(n)}^{s N}+\mathbb{F}_{i(\tau)}^{s N}$ is the net exerted force on the soil particle by DEM objects as shown by Equation 17. If the interaction does not involves a DEM object $\mathbf{F}_{i}^{N s}=0$.

Equations 4 and 5 are the expressions employed to compute the interaction forces between the two SPH phases, fluid and soil. The interaction forces for the fluid and soil, respectively, are [24, 7, 25]:

$$
\begin{aligned}
& \mathbf{a}_{i}^{f_{s}}=\sum_{j=1}^{n} m_{s} \frac{\mathbf{f}^{\text {seepage }}}{\rho_{f} \rho_{s}} W\left(r_{f s}, h\right) \\
& \mathbf{a}_{i}^{s f}=\sum_{j=1}^{n} m_{f} \frac{\mathbf{f}_{f}^{\text {seepage }}}{\rho_{f} \rho_{s}} W\left(r_{f s}, h\right)-\sum_{j=1}^{n} m_{f} \frac{p_{f}}{\rho_{f} \rho_{s}} \nabla_{i} W\left(r_{s f}, h\right)
\end{aligned}
$$

The subindex $f$ and $s$ denote fluid and soil particle, respectively. The second term in Equation 5 represents the pore fluid pressure exerted on soil particles. $\mathbf{f}^{\text {seepage }}=(\mu / k)\left(\mathbf{u}_{f}-\mathbf{u}_{s}\right)$ is the seepage force based 
on Darcy's law. $k=k_{h} \mu / \rho_{f} g$ is the intrinsic permeability, $k_{h}$ is the Darcy hydraulic conductivity (unit, $\mathrm{L} / \mathrm{T}), \mu$ and $\rho_{f}$ are the fluid viscosity and density, respectively. Dimensionally, $k$ is an area $\left(L^{2}\right)$ [26]. By using a laboratory-scale series of experiments, $[7,25,27,28]$ have demonstrated that the physics implemented in this work for the coupling of soil-water interaction forces can produce satisfying agreements with experimental data.

The equation that describes the movement of the boulders is computed using the DEM so that:

$$
m_{k} \frac{d \mathbf{u}_{k}}{d t}=m_{k} \mathbf{g}+\sum_{j=1}^{n} \mathbf{F}_{j}+\sum_{i=1}^{n} \mathbf{F}_{i}^{N p}
$$

where $m$ is the mass, $\mathbf{u}$ is the velocity, $\mathbf{g}$ the gravity, $\mathbf{F}_{j}$ is the exerted force on the $k$ DEM element by $j$ DEM particles, and $\mathbf{F}_{i}^{N p}$ is the exerted force on the DEM element by a SPH particle $i$ of any SPH phase $p$ (fluid or soil) $[29,30]$.

\subsection{Coupled SPH-DEM}

One single DEM particle will represent the DEM object, and there are not other SPH particles to represent or discretise the DEM objects. All DEM particles have a halo to avoid any "penetration" between SPH and DEM. Before starting any computation between the two methods, it is necessary to verify if the DEM

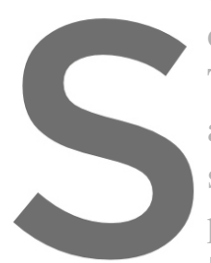
object is inside the range The main idea of this inter a DEM particle (sphere, segment, as the SPH particle (blue particle) is loca placed based on the minimum distance (Figure $1 \mathrm{~b}$ [18]. If the object is a sphere, a virtual SPH particle will be placed at the nearest point on the surface of the sphere (Figure 1a). Such virtual SPH particle will be placed as long as SPH particle is close enough

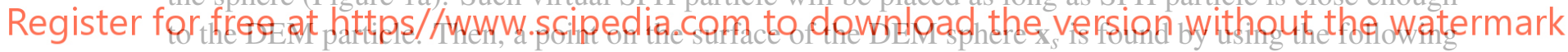
expression,

$$
\mathbf{x}_{s}=\mathbf{x}_{d e m}+r \mathbf{n}
$$

Thus, $\mathbf{x}_{s}$ gives the position of the virtual SPH particle (purple particle in Figure 1) to compute the interaction between SPH real particle (blue particle in Figure 1) and the surface of the DEM particle. $r$ is the radius of the sphere and $\mathbf{n}=\left(\mathbf{x}_{s p h}-\mathbf{x}_{d e m}\right) /\left|\mathbf{x}_{s p h}-\mathbf{x}_{d e m}\right|$ is the unit normal vector, $\mathbf{x}_{s p h}$ is the position of the SPH particle and $\mathbf{x}_{d e m}$ is the centre of the DEM object. Then, the distance $d$ between the real and virtual particle is given by Equation 8, and the overlapping distance $\delta$ between the SPH particle and the DEM halo is computed by Equation 9 (Figure 1).

$$
\begin{aligned}
d & =\left|\mathbf{x}_{s p h}-\mathbf{x}_{s}\right| \\
\delta & =\boldsymbol{\varepsilon}-d
\end{aligned}
$$


Mario Germán Trujillo-Vela, Sergio Andrés Galindo-Torres, Xue Zhang, Alfonso Mariano Ramos-Cañón and Jorge Alberto Escobar-Vargas

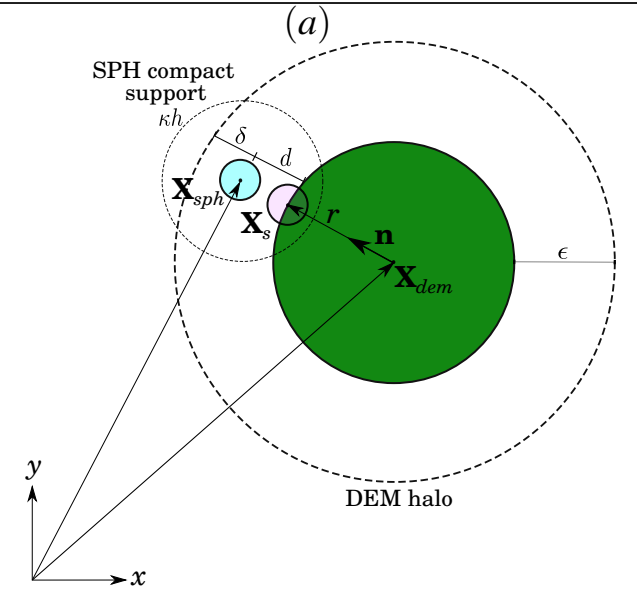

$(b)$

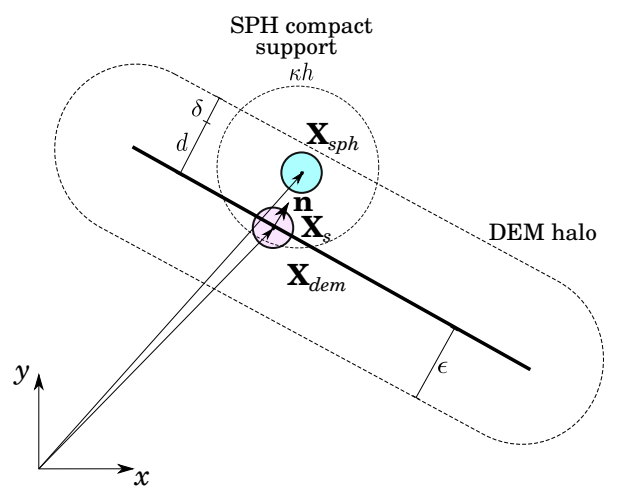

Figure 1: Coupling SPH-DEM scheme. (a) DEM sphere interacting with SPH particles. (b) DEM segments or planes are interacting with SPH particles. The blue particle represents the SPH particle, the purple particle is a virtual particle, and the circular and flat objects are DEM particles, whose positions are $\mathbf{x}_{s p h}, \mathbf{x}_{s}$, and $\mathbf{x}_{\text {dem }}$, respectively.

where $\varepsilon$ is the thickness of the halo. It has been verified that a value of the half of the initial SPH particle distribution (i.e., $\varepsilon=\Delta x / 2$ ) seems to be appropriate in this study. When the distance between the SPH particle and the DEM object surface is lower than the compact support domain (i.e., $d<k h$ ). Then, the tangential force for fluid-DEM hand, if the overlapping $\delta>0$ ), then the tangential In contrast, the normal for an elastic interaction defined as

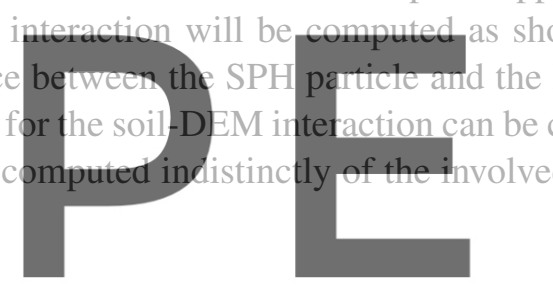

$$
\mathbf{F}_{i(n)}^{N p}=K_{n} \delta \mathbf{n}
$$

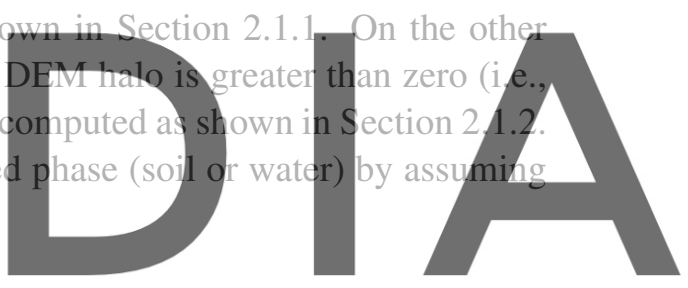

(10)

Register for free at https//www.scipedia.com to download the version without the watermark where $K_{n}$ and $\mathrm{n}$ are the normal stiffness coefficient and the normal unit vector, respectively. The constant value of the normal stiffness is computed only at the begging of the simulation as $K_{n}=0.1 \mathrm{~m}_{\min } / \Delta t^{2}$, being $m_{\min }$ the minimum value of the mass over all the particles inside the domain either SPH or DEM particles, and $\Delta t$ is the initial computational time-step. The time-step is selected as the minimum required to keep SPH particles' stability, either fluid or soil. Besides, an adaptative time-step is used for the rest of the simulation, as detailed by [25]. The normal force is dependent on the allowed penetration of the SPH particle into the DEM halo. The total force exerted on DEM objects is the summation of the force coming from all SPH particles that interact with it [29, 30]. This definition of the normal force ensures that the SPH particle does not break through the DEM particles and the normal stiffness expression guarantees the stability of the solution [31]. Now, let us define the relative velocity between the SPH particle and DEM object such as,

$$
\mathbf{u}_{r e l}=\mathbf{u}_{s p h}-\mathbf{u}_{\text {dem }}-\omega_{\text {dem }} \times\left(\mathbf{x}_{s}-\mathbf{x}_{\text {dem }}\right)
$$

where $\omega_{d e m}$ is the angular velocity of the DEM object, $\mathbf{x}_{d e m}$ is the position of the centre of the DEM 
particle and $\mathbf{x}_{s}$ is point on the surface of the DEM object (virtual SPH particle) (Figure 1). After the previous calculations, the following steps depend on what SPH material (fluid or soil) is interacting with the DEM object as it will be explained below.

\subsubsection{Fluid-solid interaction force}

The interaction term between the SPH fluid particle and DEM is defined by an extra viscous term $\mathbf{a}_{\tau}$ (Equation 12) with the same form as appear in Equation 2.

$$
\mathbf{a}_{\tau}=\frac{1}{\rho_{i}} \nabla \cdot\left(\mu_{i} \nabla \mathbf{u}_{i}\right)=\frac{4 m_{i}}{3 h^{D}} \frac{\left(2 \mu_{i}\right)}{\left(2 \rho_{i}\right)^{2}} \frac{\mathbf{u}_{r e l}}{d} \frac{\nabla_{i} W(d, h)}{W(0, h)}
$$

The expression $2 \rho_{i}$ is because the virtual particle's density equals the density of the real SPH particle $i$, and the same principle is employed with the viscosity $\mu_{i} . d$ is the distance between the real and virtual SPH particle. The additional term that multiplies Equation 12, i.e., $2 /\left[3 h^{D} W(0, h)\right]$, is used to compensate for the lack of SPH particles at the boundaries in a similar way as suggested by [10].

The total force exerted from the DEM object to the SPH particle is described as follows;

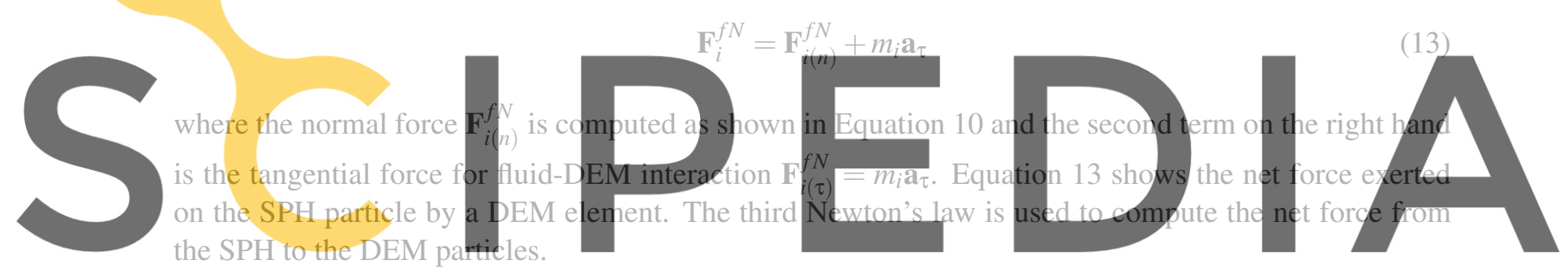

\section{Register for free at https//www.scipedia.com to download the version without the watermark}

When the soil particle is interacting with a DEM object, the normal force $\mathbf{F}_{i(n)}^{s N}$ will be computed in the same way when the interaction is fluid-DEM, Equation 10. Whereas the frictional force depends on the relative velocity and the friction coefficient. Thus, the tangential velocity is defined by Equation 14 [11], and the tangential component of the contact force acting on soil particle $i$ is computed by Equation 15 .

$$
\begin{aligned}
& \mathbf{u}_{\tau}=\mathbf{u}_{r e l}-\left(\mathbf{u}_{r e l} \cdot \mathbf{n}\right) \mathbf{n} \\
& \delta_{\tau}=\delta_{\tau}+\Delta t \mathbf{u}_{\tau} \\
& \delta_{\tau}^{*}= \begin{cases}\frac{\mu_{\phi}\left|\mathbf{F}_{n}\right|}{K_{n}} \mathbf{n}_{\tau}, & \text { if }\left|\delta_{\tau}\right|>\mu_{\phi}\left|\mathbf{F}_{n}\right| / K_{n} \\
\delta_{\tau}, & \text { otherwise }\end{cases}
\end{aligned}
$$

where $\Delta t$ is the time-step and $\delta_{\tau}$ the distance on which the SPH particle and the DEM particle are under tangential contact. The rectification of the tangential distance is given as shown by Equation 16, to ensure 
that the maximum frictional force is not exceeded. $\mu_{\phi}$ is the frictional coefficient between soil and the surface of the structure, and $\mathbf{n}_{\tau}=\delta_{\tau} /\left|\delta_{\tau}\right|$ when $\left|\delta_{\tau}\right|>0$ to avoid division by zero. The net force acting on the soil particle $i$ is given by Equation 17 .

$$
\mathbf{F}_{i}^{s N}=\mathbf{F}_{i(n)}^{s N}-K_{n} \delta_{\tau}^{*}
$$

The normal force $\mathbf{F}_{i(n)}^{s N}$ is computed as shown in Equation 10 and the second term on the right hand is the tangential force for soil-DEM interaction $\mathbf{F}_{i(\tau)}^{s N}=K_{n} \delta_{\tau}^{*}$. The net force exerted on the DEM object satisfies the third Newton's law.

\section{RESULTS OF A HYPOTHETICAL DEBRIS FLOW}

A potential application of the coupling for debris flow is here explored. It has been noticed that debris flows can drag large boulders that might have a diameter comparable to the flow depth and reach $11 \mathrm{~m}$ in diameter [32,33]. Then, large boulders are included in the mixture of soil and water to test the behaviour of having all the materials simultaneously. Thus, it is possible to test the coupling forces among all the materials in one single case, given by Equations 10,13,17. The configuration of this simulation is based on the Yangbaodi landslide presented by [34]. However, several changes were performed in the initial configuration so that it is not the intention to reproduce the Yangbaodi event. Three boulders (DEM spheres) were placed into the fluid-soil mixture, as shown in Figure 2a. The soil was assumed to be 100

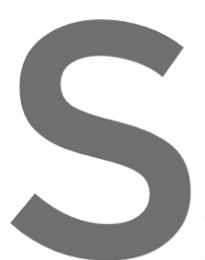
$\%$ saturated. Hence, the

and soil mass were discreti

SPH points at the beginni

simulation was $30 \mathrm{~s}$. A

overtopping problems,

in Table 1.
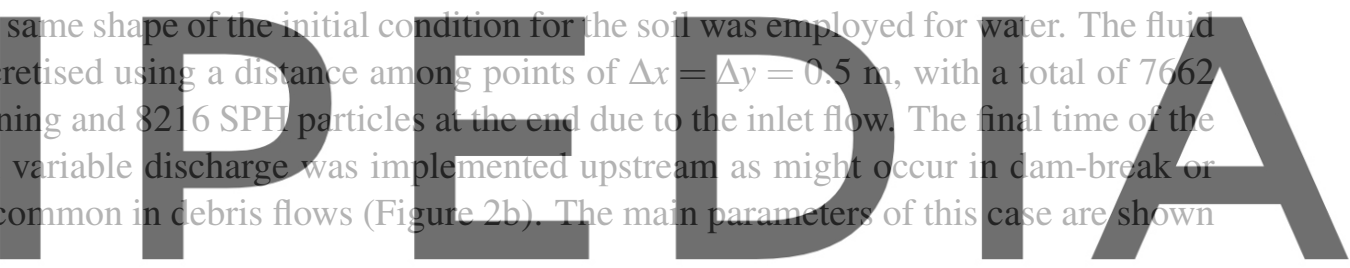

(a)

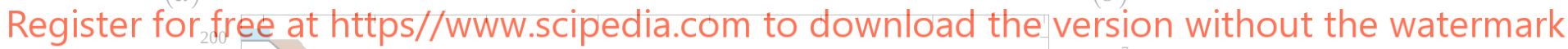
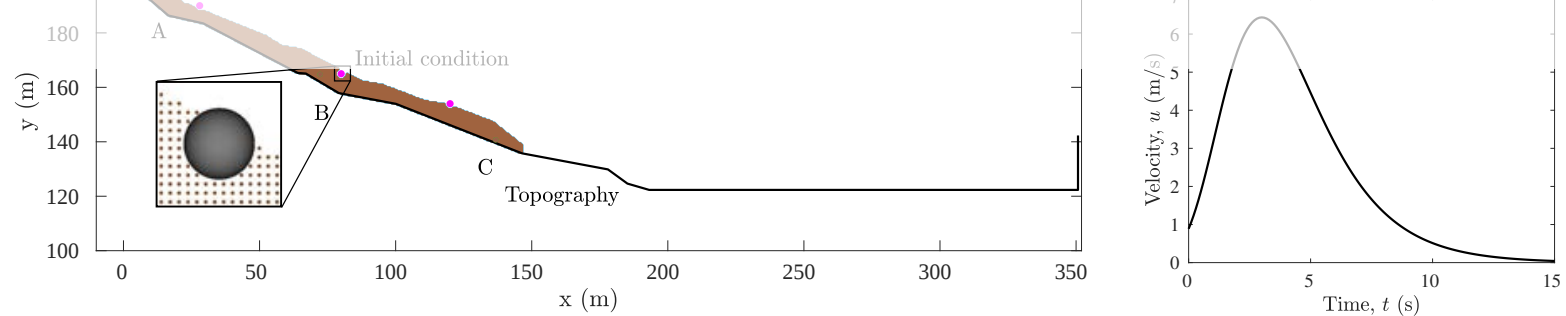

Figure 2: (a) Initial configuration of the fluid and soil SPH particles, and DEM boulders (fuchsia points). (b) Hydrograph of the inlet flow using a Gumbel shaped function.

Figure 3 shows the fluid phase as well as the boulders descending by the slope at six time-steps. The colour map shows the magnitude of the velocity of each fluid SPH particle. The velocity field in both phases have similar behaviour, and a slight difference is given in the fluid phase mainly caused by the inlet flow. The fuchsia points denote the position of each boulder at that time-step. 

Jorge Alberto Escobar-Vargas

Figure 4 presents the colour map of the pore fluid pressure at the same six-time steps. The most relevant characteristic is that the pore fluid pressure is interrupted horizontally by such big boulders. In contrast, the pore fluid pressure field seems to be more continuous in the $x$ direction when no boulders are included $[29,30]$.

Table 1: Debris flow parameters.

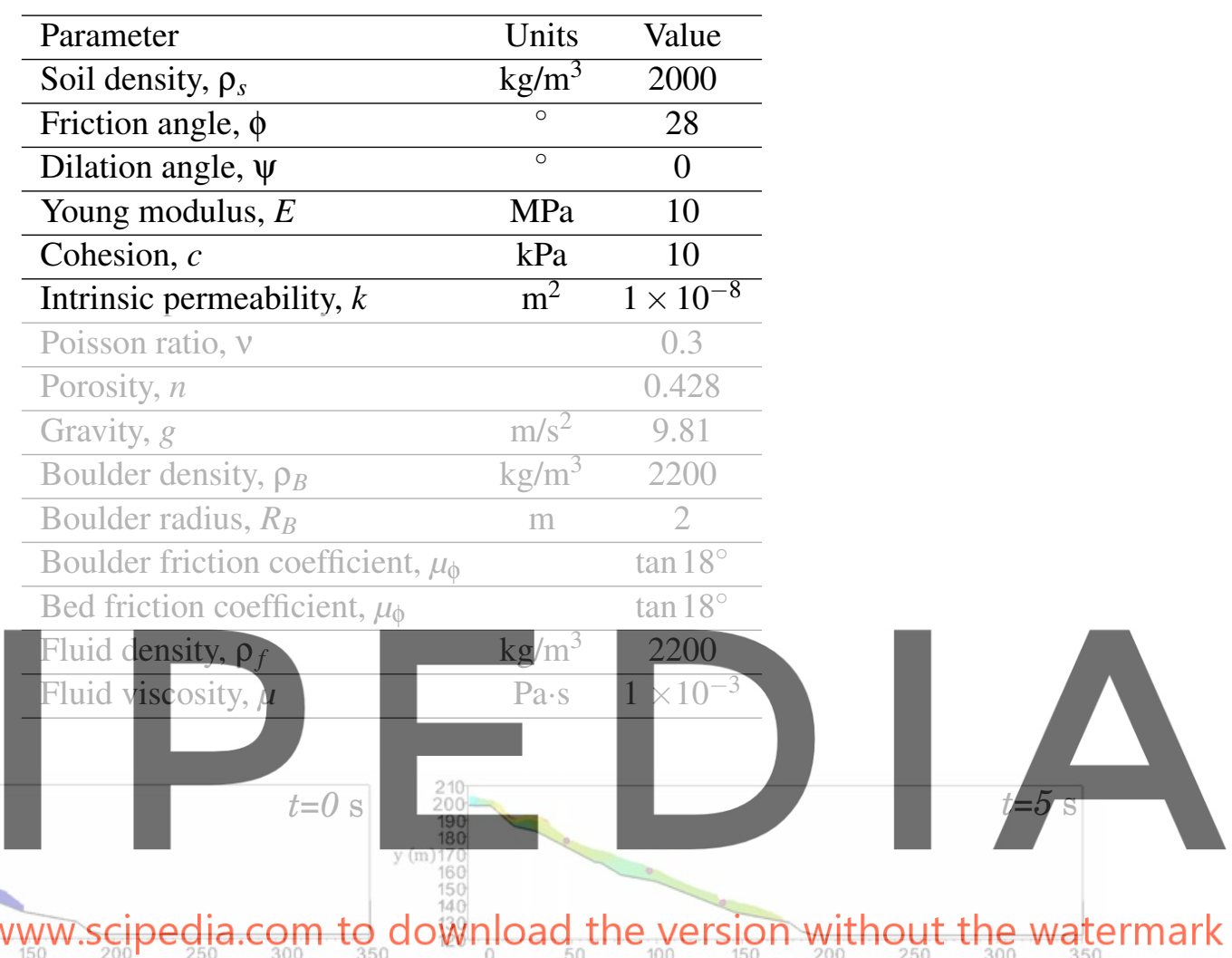

Register for free at https//www.scipedia.com to down load the version without the watermark
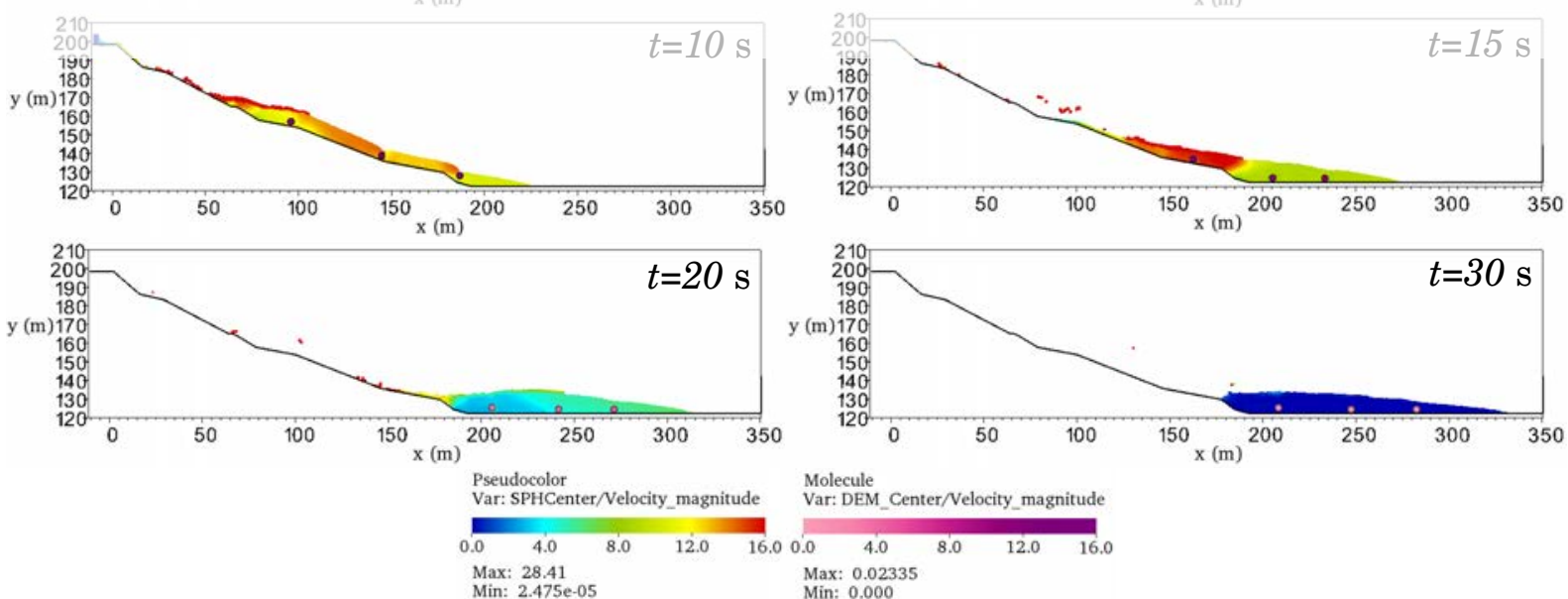

Molecule
Var: DEM_Center/Velocity_magnitude

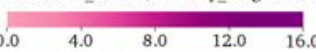
Max: 0.02335

Figure 3: Norm of the velocity vector $|\mathbf{u}|$ of SPH fluid particles and DEM boulders going downhill. 

Jorge Alberto Escobar-Vargas

The kinetic energy was checked from a control volume setup at the beginning of the horizontal zone $(x=193.1 \mathrm{~m})$ since this is the point were the mass reach the maximum velocity. Any material (soil, fluid and boulders) that was crossing the control volume, whose width was $\Delta x=0.5 \mathrm{~m}$, were added to obtain the total kinetic energy measured at that time (Figure 5a).
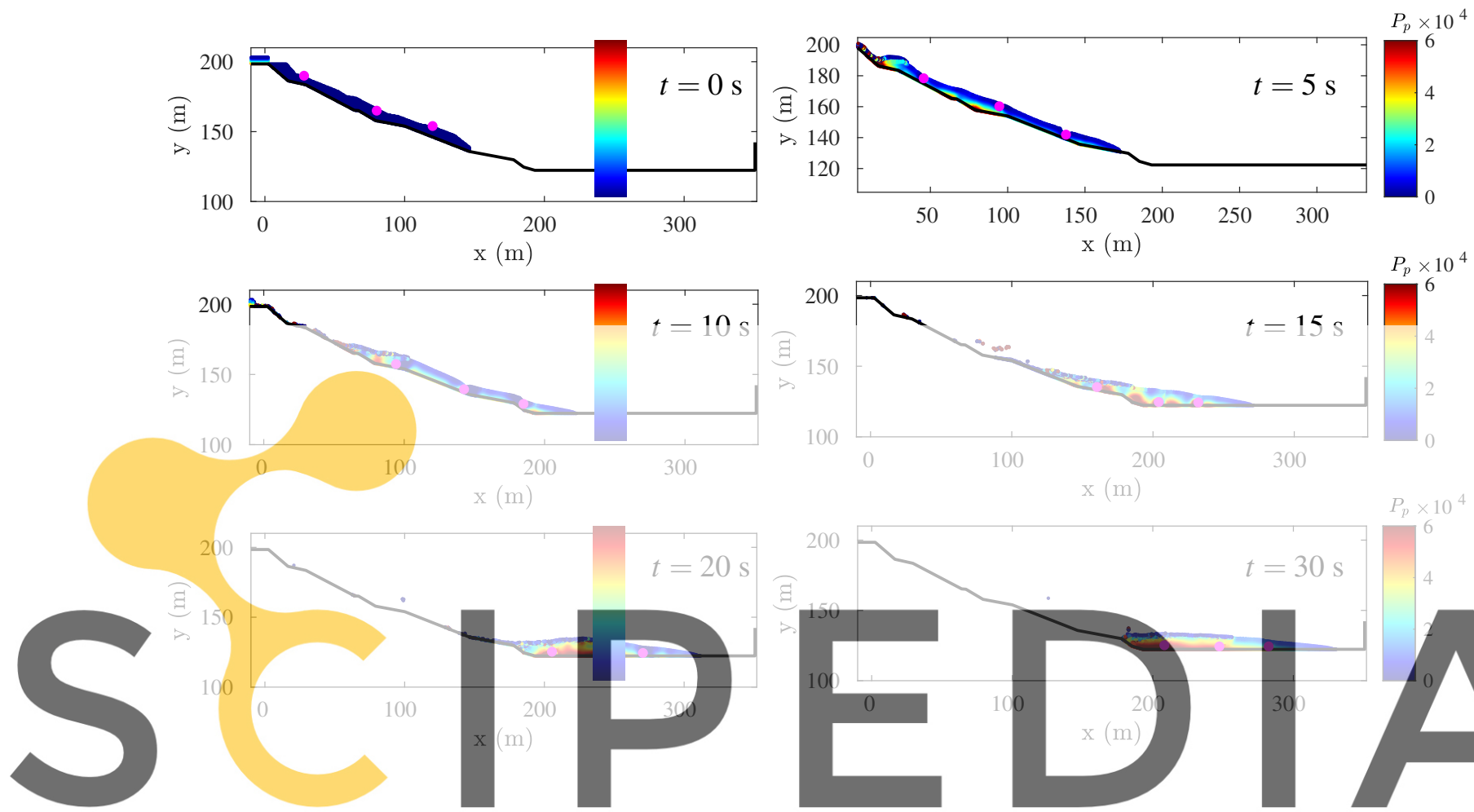

Figure 4: Pore fluid pressure during the displacement of the entire mass and boulders (fuchsia points).

\section{Register for free at https//www.scipedia.com to download the version without the watermark}

Figure $5 \mathrm{~b}$ presents the kinetic energy of the numerical solution with boulders (solid black line) and without boulders (dashed blue line) into the mixture. The simulation with no boulders is employed as a reference case to observe the importance of including large and heavy objects into the simulations when required. Figure $5 \mathrm{~b}$ shows that the average behaviour that is given by the SPH particles in both cases is similar. It is also noticeable when each boulder crosses the control volume since the kinetic energy increases five times, which is marked by the three peaks in Figure $5 b$. The boulders decrease the kinetic energy dissipation rate while they are moving, which increases the potential of damage. Such an increment is not just given by the mass of the boulder taken into account in the control volume, but by their velocities; as one is zero, there is not kinetic energy coming from DEM spheres. [35] have demonstrated that the force coefficient, which is employed to estimate the impact force on rigid barriers, is strongly dependent on both the diameter of the particles and the Froude number of the flow. Larger particles have a higher force coefficient, increasing the impact force. The quantitative estimation of the energy and force in such phenomena is essential to consider the damage level of a specific structure or provide data for designing the retention structures. 

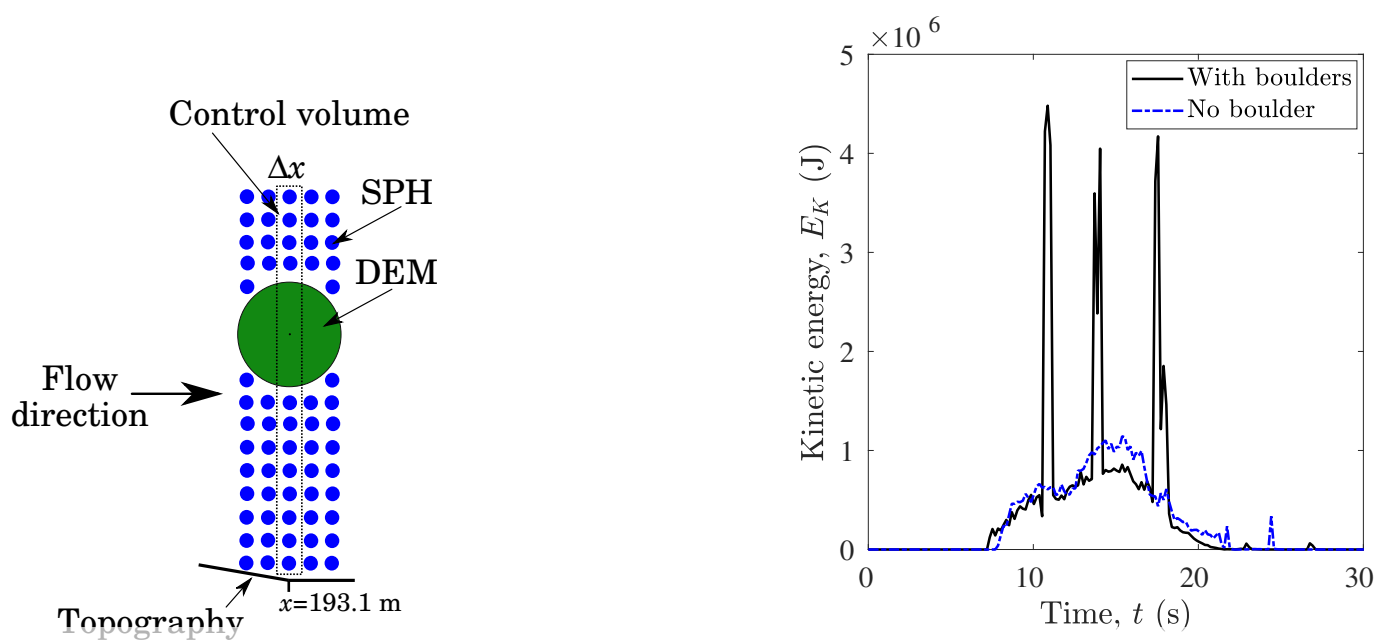

Figure 5: (a) Scheme of the control volume to measure the kinetic energy as a function of time. (b) Measurement of the kinetic energy at the distance of $x=193.1 \mathrm{~m}$ for the entire depth of the flow with boulders (black line) and with no boulders (blue line).

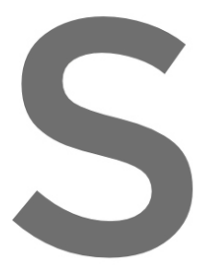

4 CONCLUSIONS

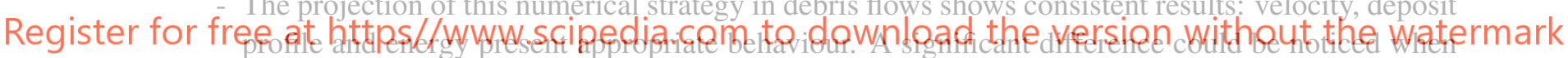

large boulders are crossing the control volume as well as the soil and fluid materials, which can contribute to structure design of retaining barrier for debris fiows. However, this requires validation with field data.

\section{REFERENCES}

[1] Dai, Z.; Huang, Y.; Cheng, H. and Xu, Q. Sph model for fluid-structure interaction and its application to debris flow impact estimation. Landslides. (2017) 14(3):917-928.

[2] Leonardi, A.; Wittel, F.K.; Mendoza, M.; Vetter, R; and Herrmann, H.J. Particlefluidstructure interaction for debris flow impact on flexible barriers. Comput-Aided Civ Inf. (2016) 31:323-333.

[3] Bui, H.H.; Fukagawa, R.; Sako, K. and Ohno, S. Lagrangian meshfree particles method (sph) for large deformation and failure flows of geomaterial using elastic-plastic soil constitutive model. Int J Numer Anal Methods Geomech. (2008) 32(12):1537-1570.

[4] Peng, C.; Guo, X.; Wu, W. and Wang, Y. Unified modelling of granular media with smoothed 
particle hydrodynamics. Acta Geotech. (2016) 11(6):1231-1247.

[5] Abdelrazek, A.M.; Kimura, I. and Shimizu, Y. Simulation of three-dimensional rapid freesurface granular flow past different types of obstructions using the sph method. J Glaciol. (2016) 62(232):335-347.

[6] Nguyen, C.T.; Nguyen, C.T.; Bui, H.H.; Nguyen, G.D. and Fukagawa, R. A new sph-based approach to simulation of granular flows using viscous damping and stress regularisation. Landslides. (2017) 14(1):69-81.

[7] Korzani, M.G.; Galindo-Torres, S.A.; Scheuermann, A. and Williams, D.J. Smoothed particle hydrodynamics for investigating hydraulic and mechanical behaviour of an embankment under action of flooding and overburden loads. Comput Geotech. (2018) 94:31-45.

[8] Potapov, A.V.; Hunt, M.L. and Campbell, C.S. Liquid-solid flows using smoothed particle hydrodynamics and the discrete element method. Powder Technol. (2001) 116(2-3):204-213.

[9] Cui, Y.; Nouri, A.; Chan, D. and Rahmati, E. A new approach to dem simulation of sand production. J Petrol Sci Eng. (2016) 147:56-67.

[10] Nassauer, B.; Liedke, T. and Kuna, M. Development of a coupled discrete element (dem)-smoothed particle hydrodynamics (sph) simulation method for polyhedral particles. Computational Particle Mechanics. (2016) 3(1):95-106.

[11] Wang, J. and Chan, D. Frictional contact algorithms in sph for the simulation of soil-structure interaction. Int J Numer Anal Methods Geomech. (2014) 38(7):747-770.

[12] Cundall, P.A. and Strack, O.DL. A discrete numerical model for granular assemblies. Geotechnique. (1979) 29(1):47-65.

[13] Galindo-Torres, S.A. A coupled discrete element lattice boltzmann method for the simulation of fluid-solid interaction with particles of general shapes. Comput. Methods Appl. Mech. Engrg. (2013) 265:107-119.

[14] Tan, H. and Chen, S. A hybrid dem-sph model for deformable landslide and its generated surge waves. Adv Water Resour. (2017) 108:256-276.

[15] Xu, W.J.; Yao, Z.G.; Luo, Y.T. and Dong, X.Y. Study on landslide-induced wave disasters using a 3D coupled SPH-DEM method. Bull. Eng. Geol. Environ. (2020) 79(1):467-483.

[16] Liu, C.; Sun, Q. and Zhou, G.G.D. Coupling of material point method and discrete element method for granular flows impacting simulations. Int J Numer Methods Eng. (2018) 115(2):172-188.

[17] Li, X and Zhao, J. A unified cfd-dem approach for modeling of debris flow impacts on flexible barriers: A unified cfd-dem approach for modeling of debris flow impacts on flexible barriers. Int J Numer Anal Methods Geomech. (2018) 42(14):1643-1670.

[18] Galindo-Torres, S.A. and Pedroso, D.M. Molecular dynamics simulations of complex-shaped particles using voronoi-based spheropolyhedra. Physical Review E. (2010) 81(6):061303.

[19] Morris, J.P. Simulating surface tension with smoothed particle hydrodynamics. Int J Numer Methods Fluids. (2000) 33(3):333-353.

[20] Molteni, D. and Colagrossi, A. A simple procedure to improve the pressure evaluation in hydrody- 
namic context using the SPH. Computer Physics Communications. (2009) 180(6):861-872.

[21] Morris, J.P.; Fox, P.J. and Zhu, Y. Modeling low Reynolds number incompressible flows using sph. J. Comput. Phys.. (1997) 136(1):214-226.

[22] Cleary, P.W. and Monaghan, J.J. Conduction modelling using smoothed particle hydrodynamics. J. Comput. Phys.. (1999) 148(1):227-264.

[23] Monaghan, J.J. Sph without a tensile instability. J. Comput. Phys. . (2000) 159(2):290-311.

[24] Bui, H.H. and Nguyen, G.D. A coupled fluid-solid sph approach to modelling flow through deformable porous media. Int J Solids Struct. (2017) 125:244-264.

[25] Korzani, M.G.; Galindo-Torres, S.A.; Scheuermann, A. and Williams, D.J. Sph approach for simulating hydro-mechanical processes with large deformations and variable permeabilities. Acta Geotech. (2018) 13(2):303-316.

[26] Kirkham, M.B. Principles of soil and plant water relations. Academic Press, 2nd edition, (2014).

[27] Islam, M.R.; Hayano, K. and Rahman, Md.A. Insights into effects of seepage on failure of breakwater mound: Experimental and numerical investigations. Indian Geotechnical Journal. (2019) 49(5):531-542.

[28] Islam, M.R.; Rahman, Md.A. and Hayano, K. Application of smoothed particle hydrodynamics (sph) for simulating various geotechnical problems. SN Applied Sciences. (2020) 2(4):1-14.

[29] Trujillo-Vela, M.G.; Galindo-Torres, S.A.; Zhang, X.; Ramos-Cañón, A.M. and Escobar-Vargas, J.A. Smooth particle hydrodynamics and discrete element method coupling scheme for the simulation of debris flows. Comput and Geotech. (2021) 125:103669.

[30] Trujillo-Vela, M.G. Numerical modelling of debris flows with large boulders. $\mathrm{PhD}$ thesis, Pontificia Universidad Javeriana, Bogotá D.C., Colombia, (2021).

[31] Wang, J.; Wu, H.; Gu, C.S. and Hua, H. Simulating frictional contact in smoothed particle hydrodynamics. Sci. China Technol. Sci.. (2013) 56(7):1779-1789.

[32] Larsen, M.C.; Wieczorek, G.F., Eaton, L.S.; Morgan, B.A. and Torres-Sierra, H. Natural hazards on alluvial fans; the venezuela debris flow and flash flood disaster. Technical Report 103-01, USGS, (2002).

[33] Takahashi, T. Debris flow: mechanics, prediction and countermeasures. CRC press, (2014).

[34] Zhang, X.; Krabbenhoft, K.; Sheng, D. and Li, W. Numerical simulation of a flow-like landslide using the particle finite element method. Comput. Mech. (2015) 55(1):167-177.

[35] Cui, Y.; Choi, C.E.; Liu, L.HD. and Ng, C.WW. Effects of particle size of mono-disperse granular flows impacting a rigid barrier. Nat Hazards. (2018) 91(3):1179-1201. 